\title{
On Representations of Some Thickness-Two Graphs
}

\author{
Extended Abstract
}

\author{
Joan P. Hutchinson \\ Department of Mathematics \\ Macalester College \\ St. Paul, MN 55105, U.S.A. \\ hutchinson@macalstr.edu
}

\author{
Thomas Shermer \\ Department of Computer Science \\ Simon Fraser University \\ Burnaby, BC, Canada \\ shermer@cs.sfu.ca
}

\author{
Andrew Vince \\ Department of Mathematics \\ University of Florida \\ Gainesville, FL 32611, U.S.A. \\ vince@math.ufl.edu
}

\begin{abstract}
This paper considers representations of graphs as rectanglevisibility graphs and as doubly linear graphs. These are, respectively, graphs whose vertices are isothetic rectangles in the plane with adjacency determined by horizontal and vertical visibility, and graphs that can be drawn as the union of two straight-edged planar graphs. We prove that these graphs have, with $n$ vertices, at most $6 n-20$ (resp., $6 n-18$ ) edges, and we provide examples of these graphs with $6 n-20$ edges for each $n \geq 8$.
\end{abstract}

\section{Introduction}

A thickness-two graph $G$ is one whose edge set can be partitioned into two planar graphs, each on one copy of the vertex set of $G$. These graphs are of theoretical interest and arise in a multitude of applications. For example, it is an NPcomplete problem to determine whether a graph has thickness two [9], and the upper bound on their chromatic number is known only to lie between 9 and $12[12,4,6]$. These graphs arise in models for printed circuit boards [5, 6] and in VLSI design and layout [18] in which all connections are either horizontal or vertical and so divide naturally into two planar layers. Visibility representation of these graphs is of increasing interest; see, for example, [11, chap.7].

We study thickness-two graphs and their representations (and nonrepresentations) as rectangle-visibility graphs and as doubly linear graphs. We show that not all thickness-two graphs have these representations. Specifically we show that the most (edge) dense thickness-two graphs are neither rectangle-visibility nor doubly linear graphs, though these graph representations are ubiquitous among thickness-two graphs of lower density.

A bar-visibility graph $[8,19]$ is one whose vertices can each be represented by a closed horizontal line segment in the plane, having pairwise disjoint relative interiors, with two vertices adjacent in the graph if and only if the corresponding segments are vertically visible. Two segments are considered vertically visible when there is a nondegenerate rectangle $R$ such that $R$ intersects only these two segments and the horizontal sides of $R$ are subsets of these two segments. (For variations on 
this definition, see $[11,14]$.) Clearly a bar-visibility graph is planar. Not all planar graphs are bar-visibility graphs since the latter are characterized as those planar graphs for which there is a planar embedding with all cut vertices on a common face [14, 19].

A natural two-dimensional analogue is that of a rectangle-visibility graph, a graph whose vertices can each be represented by a closed rectangle in the plane with sides parallel to the axes, having pairwise disjoint interiors, with two vertices adjacent in the graph if and only if the corresponding rectangles are vertically or horizontally visible (with horizontal visibility defined analogously to vertical). Note that the bands of visibility may overlap and cross. Every planar graph is a rectanglevisibility graph [8], and it is clear that every rectangle-visibility graph has thickness at most two. Even more, a rectangle-visibility graph is the union of two barvisibility graphs. Our main result on these graphs is that a rectangle-visibility graph with $n$ vertices has at most $6 n-20$ edges, as distinguished from thickness-two graphs which have at most $6 n-12$ edges. (The latter fact follows from Euler's formula for planar graphs, which implies that a planar graph with $n$ vertices has at most $3 n-6$ edges.) Thickness-two graphs can have as many as $6 n-12$ edges; we show also that for every $n>7$ there is a rectangle-visibility graph with $6 n-20$ edges. Using similar methods, in [2,3] it is proved that a bipartite rectangle-visibility graph has at most $4 n-12$ edges.

It is a consequence of a classical theorem of Steinitz on polyhedra (see [13]) that every planar graph $\mathrm{G}$ has a linear or straight-line embedding in the plane. This means that

(1) every edge is a straight line segment,

(2) no vertex lies in the interior of an edge, and

(3) edges do not cross.

(Results on rectilinear drawings, which satisfy only (1) and (2), are obtained in [15].) If, instead of property (3), we require that

(3') G can be partitioned into two subgraphs, each without edge crossings,

then $\mathrm{G}$ is called doubly linear. Again it is clear that doubly linear graphs have thickness at most two. We prove that a doubly linear graph with $n$ vertices has at most $6 n-18$ edges, and for each $n>7$ we have an example of a doubly linear graph with $6 n-20$ edges, two edges less than our upper bound. We give examples of doubly linear graphs that are not rectangle-visibility graphs and conjecture that every rectangle-visibility graph is doubly linear.

Since it is known that the problem of recognizing thickness-two graphs is NP-complete, it may be difficult to obtain a complete characterization of rectanglevisibility or of doubly linear graphs. These concepts come from $[5,8]$, and from the Workshop on Visibility Representations, McGill University Bellairs Research Institute, held in February, 1993. Complete details of this work are included in [7].

\section{Examples of rectangular and doubly linear representations}

Some examples of these representations are shown in the figures. Figures 1 and 2 show a rectangle-visibility and a doubly linear representation, respectively, of the complete graph $\mathrm{K}_{8}$. 


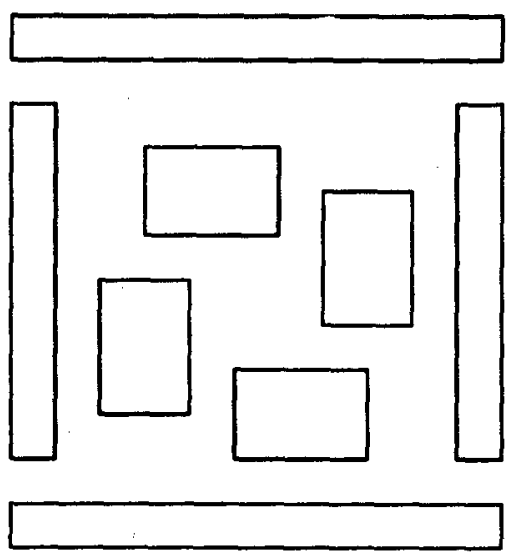

Fig. 1. A rectangle-visibility representation of $\mathrm{K}_{\mathbf{8}}$.

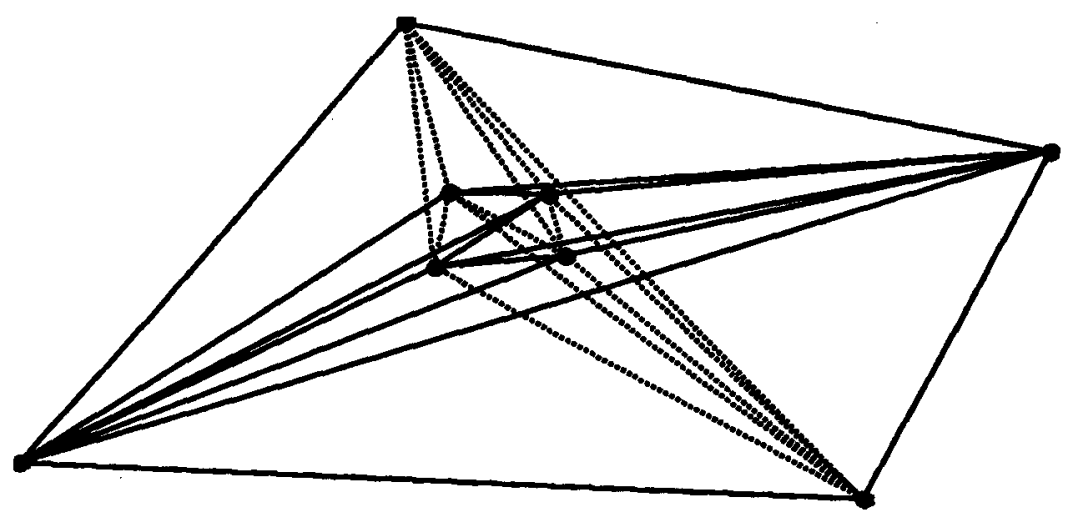

Fig. 2. A doubly linear representation of $\mathrm{K}_{\mathbf{8}}$.

This is the largest complete graph so representable since $\mathrm{K}_{\mathbf{9}}$ has thickness three $[1,16]$. It is not hard to add another vertex, adjacent to six others, to each of these representations to obtain $\mathrm{K}_{9}$ minus two edges; it can be arranged for these missing edges to be either mutually incident or nonincident. These graphs and $K_{8}$ have $6 n-20$ edges, $n=9,8$, respectively. $K_{9}$ minus one edge $\left(K_{9}-e\right)$ has thickness two with $6 n-19$ edges [17]. Theorem 3 will show that $K_{9}-e$ is therefore not a rectangle-visibility graph, though it is the union of two bar-visibility graphs. We conjecture that $\mathrm{K}_{9}-\mathrm{e}$ is not doubly linear.

Figure 3 shows a rectangular representation of $K_{5,5}$ plus four edges, and Figure 4 shows a closely related doubly linear representation of $K_{5,5}$. Figure 3 can be extended to a rectangular representation of $\mathrm{K}_{5,6}$ plus edges by adding a long rectangle along the left side, and Figure 4 can be similarly extended to a doubly linear representation of $K_{5,6}$. In [2,3] it is shown that $K_{p, q}$ with $p$ and $q$ at least 5 is not a rectangle-visibility graph (and that $\mathrm{K}_{5,5}$ minus an edge and $\mathrm{K}_{5,5}$ plus an edge are rectangle-visibility graphs). Thus $\mathrm{K}_{5,5}$ and $\mathrm{K}_{5,6}$ are doubly linear graphs, but not 
rectangle-visibility graphs. These examples point up an essential difference between the two classes of graphs: namely, that although a subgraph of a doubly linear graph is also doubly linear, the same is not true for rectangle-visibility graphs.

OPEN QUESTION. Is there a rectangle-visibility graph that is not doubly linear?

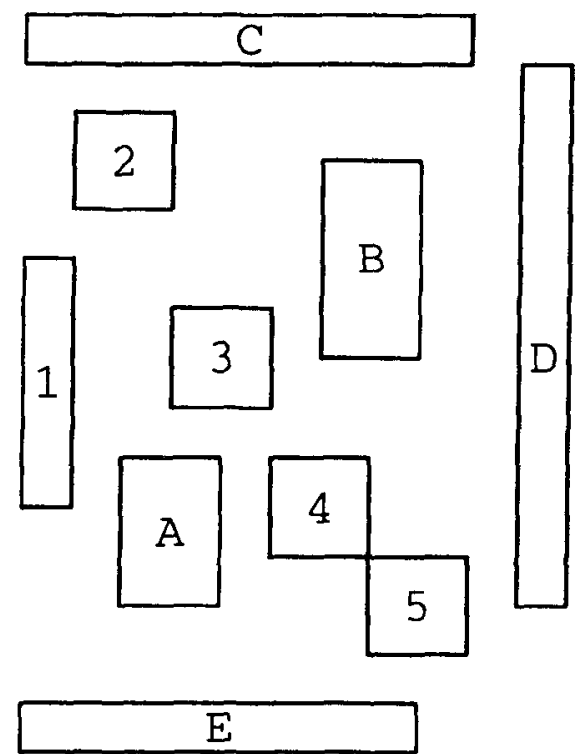

Figure 3. A rectangle-visibility representation of $\mathrm{K}_{5,5}$ plus four edges.

It is not difficult to obtain, for every $n$, a rectangle-visibility and a doubly linear representation of the join of $K_{4}$ and $P_{n}$ and the join of $K_{4}$ and $C_{n}$, where $P_{n}$ and $\mathrm{C}_{\mathrm{n}}$ are, respectively, the path and the cycle on $\mathrm{n}$ vertices. The join of two disjoint graphs $G$ and $H$ is the disjoint union of these two graphs together with an edge joining vertices $g$ and $h$, for each vertex $g$ of $G$ and vertex $h$ of $H$, and is denoted $\mathrm{G}+\mathrm{H}$. In these examples $\mathrm{K}_{4}$ cannot be replaced by $K_{5}$ for $n>12$ since these graphs would contain $\mathrm{K}_{5,13}$ which, by Euler's formula, has thickness at least 3 .

In [7] we prove the following two propositions on representations of joins. Proposition 1 gives another family of graphs that have a rectangular representation, and Proposition 2 proves these are also doubly linear. Note that, as long as G contains a cycle, $P_{2}+G$ is not planar since it contains a homeomorph of $K_{5}$.

PROPOSITION 1. If $\mathrm{G}$ is a 2-connected planar graph or, more generally, a barvisibility graph, then $P_{2}+G$ is a rectangle-visibility graph. 


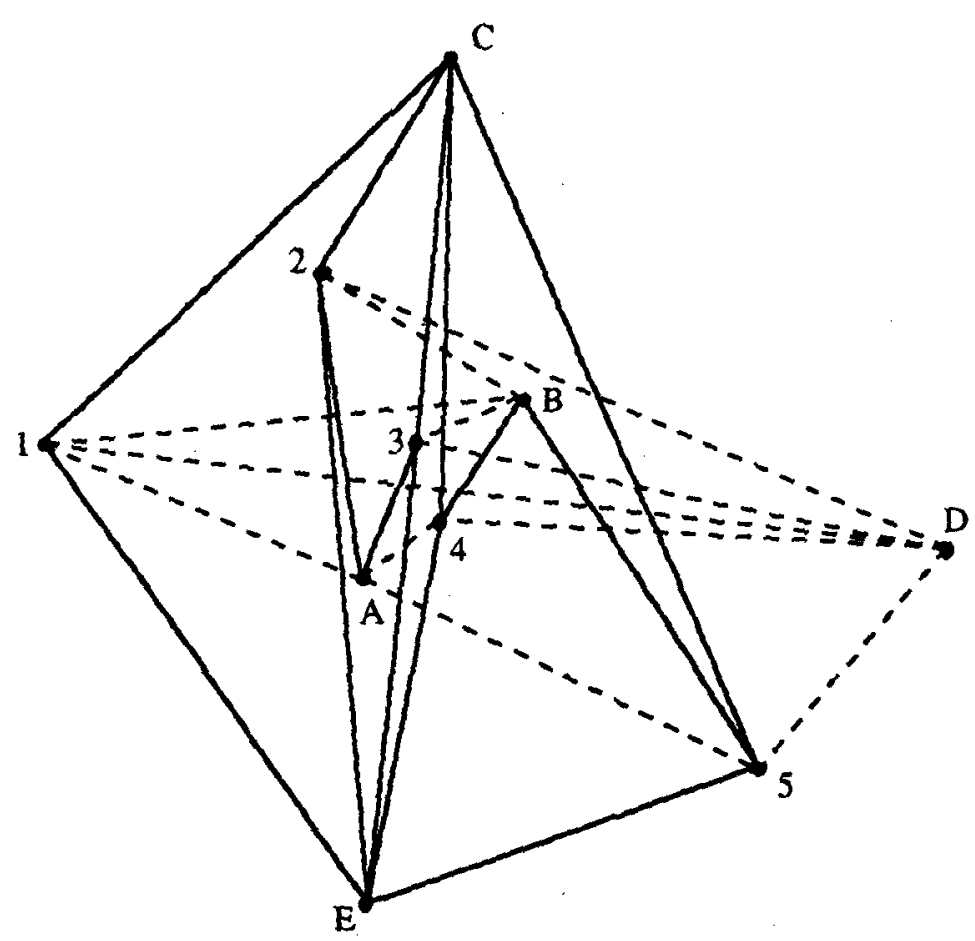

Fig. 4, A doubly linear representation of $\mathrm{K}_{5,5}$.

PROPOSITION 2. If $G$ is a planar graph, then $P_{2}+G$ is doubly linear.

Additional rectangular examples are found in Figures 5 and 6 , and related dou linear graphs are described in a sketch of the proof of Theorem 8.

\section{Edge bounds and densities for rectangle-visibility grap}

In [7] we determine a tight bound, $6 n-20$, on the maximum number edges in a rectangle-visibility graph with $n$ vertices. In addition we examine possible edge-densities for rectangle-visibility graphs and for graphs that do not hi a rectangle-visibility representation. More precisely, if $m$, the number of edges greater than $6 n-20$, then no rectangle-visibility representation is possible: essentially all $m \leq 6 n-20$ there exist both examples of graphs that have a rectang visibility representation and examples of graphs that do not. In particular, we give example of a rectangle-visibility graph with $n$ vertices and $m$ edges for each $m w$ $0 \leq m \leq 6 n-20$ and an example of a connected rectangle-visibility graph for each with $n-1 \leq m \leq 6 n-20$ (except for two pairs $(m, n)$ ).

THEOREM 3. A rectangle-visibility graph on $n \geq 5$ vertices has at most $6 n-$ edges. 
SKETCH OF PROOF. Let $G$ be a rectangle-visibility graph with representation $R^{*}$. Let $R$ be a rectangle in $R^{*}$ and define $N(R)$ to be the set of rectangles in $R^{*}$ that intersect with positive area the one-way infinite band of all points "north" of $R$. Select $R_{1}$ to be a rectangle $R$ with $N(R)$ empty and with the greatest $y$-coordinate for its bottom. Note that if $R^{\prime}$ is visible to $R_{1}$ horizontally, then $N\left(R^{\prime}\right)$ is empty; otherwise there is another rectangle with $N(R)$ empty and $y$-coordinate larger than $R_{1}$ 's for its bottom. Move $R_{1}$ northward, above the rest of the configuration, and expand it horizontally until it is wider than the whole representation. The new $R_{1}$ has retained all its previous visibilities and may have gained some.

We repeat this process of rectangle movement and expansion to the south, east, and west. The resulting configuration is bordered by four rectangles as in Figure 1. The resulting rectangle-visibility graph $G^{\prime}$ contains $G$ as a subgraph. Using Euler's formula for planar graphs, upper bounds on the number of horizontal and vertical edges of $G^{\prime}$ are $3 n-12$ and $3 n-8$, respectively, because the topmost and bottommost rectangles of $G^{\prime}$ have horizontal degree zero and the rightmost and leftmost rectangles have vertical degree two. Thus $G$ has at most $6 n-20$ edges. QED

COROLLARY 4. Let $\mathrm{G}^{\prime}$ with $n^{\prime} \geq 5$ vertices be a subgraph of a rectangle-visibility graph $G$. Then $G^{\prime}$ has at most $6 n^{\prime}-20$ edges.

See [3] for similar proofs that a bipartite rectangle-visibility graph and a bipartite subgraph of a rectangle-visibility graph with $n$ vertices have at most $4 n-12$ edges. Bipartite rectangle-visibility examples with at most $4 n-16$ edges are given also for each $n \geq 7$, and for each $n \geq 16$ bipartite graphs with $n$ vertices and $4 n-12$ edges are known that are subgraphs of rectangle-visibility graphs [10].

We also show that the bound of Theorem 3 is best possible for all $n \geq 8$. (For $\mathrm{n} \leq 8$, as noted before, the complete graphs give the best possible bound.) Figures 5 and 6 show rectangular representations with $6 n-20$ edges and $n=16$ and 17 vertices, respectively, and Theorem 5 states that similar graphs can be constructed for all $\mathbf{n} \geq 8$.

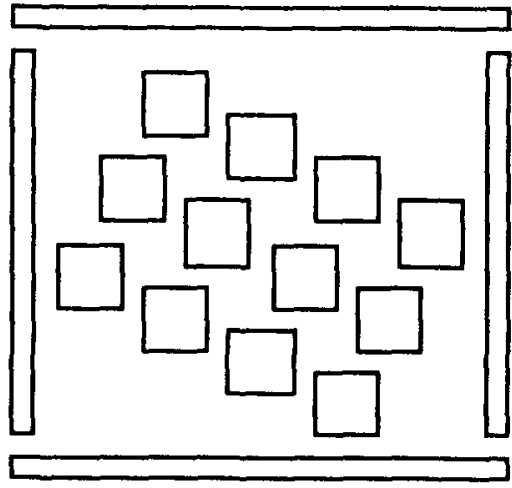

Fig. 5. A rectangular representation with $n=3.4+4$.

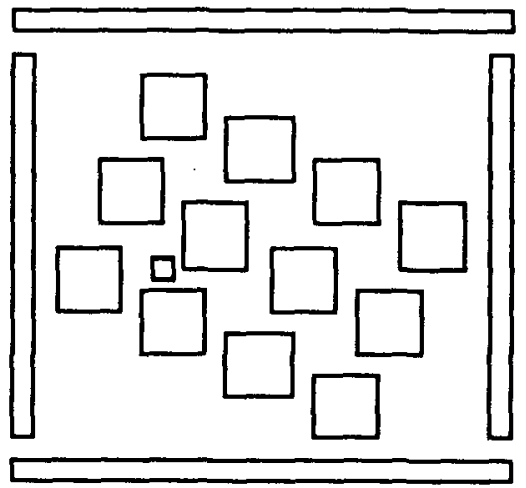

Fig. 6. A rectangular representation with $n=13+4$. 
THEOREM 5. There is a rectangle-visibility graph with $n$ vertices and $6 n-20$ edges for each $n \geq 8$. next result.

Rectangle-visibility graphs with fewer edges are also possible, as seen in the

THEOREM 6. With the exception of the cases $(n, m)=(6,16)$ and $(n, m)=(7,22)$, the following holds for all $n \geq 4$ :

(a) For each $m$ with $0 \leq m \leq 6 n-20$, there is a rectangle-visibility graph with $n$ vertices and $m$ edges.

(b) For each $m$ with $n-1 \leq m \leq 6 n-20$, there is a connected rectangle-visibility graph with $n$ vertices and $m$ edges.

The exceptions arise since a simple graph with $n$ vertices has at most $n(n-1) / 2$ edges. Otherwise these graphs are constructed from those of Theorem 5 unioned either with a graph with no edges or with a path (for the connected case.)

Families of graphs with $n^{\prime} \geq 9$ vertices and $m^{\prime} \geq 35$ edges that are not representable by rectangles can also be found. For any $0 \leq m \leq 6 n-20$ for which there is a rectangle-visibility graph $\mathrm{G}$ with $\mathbf{n}$ vertices and $\mathrm{m}$ edges, form the disjoint union of $G$ with $K_{9}-e$ to obtain a graph with $m+35$ edges and $n+9$ vertices. By Corollary 4 , the new graph is not a rectangle-visibility graph since $\mathrm{K}_{\mathbf{9}}$-e has more than $6 n-20, n=9$, edges. Connected graphs $G$ together with $K_{9}-e$ plus an adjoining edge similarly give connected examples.

\section{Edge bounds and densities for doubly linear graphs}

We also present parallel results for doubly linear graphs; however, our examples, related to those of Theorem 5 and having $6 n-20$ edges, come only within two of the edge-bound $6 n-18$ of Theorem 7 .

THEOREM 7. If $G$ is a doubly linear graph with $n \geq 4$ vertices, then $G$ has at most $6 n-18$ edges.

In fact, for $n>4$, the proof establishes an edge-bound of $6 n-20$ except in the case when the convex hull of the embedding consists of three vertices. Except for $K_{4}$, we have no example of a doubly linear graph with more than $6 n-20$ edges. The graphs of the next result are doubly linear analogues of those of Theorem 5.

THEOREM 8. There is a doubly linear graph with $n$ vertices and $6 n-20$ edges for each $n \geq 8$.

SKETCH OF THE PROOF. Suppose that $n=k m+4$ with $k, m>1, m \leq k$, and let $q \geq 2$ be an integer such that $k \leq q m$. Let $S$ be the grid points in the rectangle

$$
T=\{(x, y): 0 \leq x \leq k-1,0 \leq y \leq m-1\},
$$


and let

$$
a=(-4 k,-m), b=(4 k, 3 m), c=(8 k, 3 m-10 q m) \text {, and } d=(-k, 3 m+13 q m) .
$$

The vertices $a, b, c$, and $d$ are joined by straight edges to form a $K_{4}$ with no edge intersecting the rectangle $T$.

We form two sets $\mathrm{R}$ and $\mathrm{B}$ of edges, each a linear triangulation. Let $\mathrm{R}$ have the edges

$\{s, s+(1,0)\},\{s, s+(1,1)\},\{s, s+(2,1)\}$ for all $s$ in $S$ (and when the second vertex is an element of $S)$;

$\{a,(x, y)\}$ for $x=0$ or $x=1$ or $y=0$;

$\{b,(x, y)\}$ for $x=k-1$ or $x=k-2$ or $y=m-1$;

the edges of the $\mathrm{K}_{4}$ formed by $\mathrm{a}, \mathrm{b}, \mathrm{c}$, and $\mathrm{d}$; and

$\{c,(k-1,0)\}$.

Thus $\mathrm{a}$ is connected to the left two columns of vertices and to the bottom vertices of $\mathrm{T}$, and $\mathrm{b}$ is connected to those on the top and in the two rightmost columns.

Let $B$ have the edges

$\{s, x+(0,1)\},\{s, s+(-1,1)\},\{s, s+(-1,2)\}$ for all $s$ in $S$ (and when the second vertex is in $S$ );

$\{c,(x, y)\}$ for $x=k-1$ or $y=0$ or $y=1$;

$\{d,(x, y)\}$ for $x=0$ or $y=m-1$ or $y=m-2$;

the edges of the $\mathrm{K}_{4}$ except for the edge $\mathrm{ab}$;

$\{a,(0,0)\}$, and $\{b,(k-1, m-1)\}$.

Thus $\mathrm{c}$ is connected to the bottom and to the right of the rectangle by straight edges (since $q \geq 2$ ), and $d$ is connected to the left and to the top vertices.

It is then a routine set of slope calculations and edge counts to see that this gives a doubly linear graph with $6 n-20$ edges.

QED

Since a subgraph of a doubly linear graph is doubly linear, we can achieve in a graph with $n$ vertices any desired number of edges less than $6 n-20$. To construct families of non-doubly linear graphs one can begin as before with a specific graph that is not doubly linear and form the union with a doubly linear graph of any desired size. For example, one can begin with $\mathrm{K}_{9}$, which is not doubly linear since it has thickness three. Or one can begin with $\mathrm{K}_{12}-\mathrm{F}$, the complete graph on 12 vertices minus a one-factor. Let $G_{1}$ be the graph of the icosahedron, and let $G_{2}$ be the graph on the same set of vertices with vertices adjacent if they are at distance two in $\mathrm{G}_{1}$. In fact, $G_{1}$ and $G_{2}$ are isomorphic, and their union is $K_{12}-F$, showing the latter to have thickness two. However, $K_{12}-F$ has 12 vertices and $60=6 n-12$ edges, and so by Theorem 7 is not doubly linear.

ACKNOWLEDGMENTS. The authors would like to thank the participants in the Workshop on Visibility Representations, held in February, 1993, at the McGill University Bellairs Research Institute, Barbados, and especially the other workshop organizer, S. H. Whitesides, for stimulating questions and answers. 


\section{References}

1. J. Battle, F. Harary, and Y. Kodama, Every planar graph with nine points has a nonplanar complement, Bull. Amer. Math. Soc. 68 (1962) 569-571.

2. A. Dean and J. P. Hutchinson, Rectangle-visibility representations of bipartite graphs, Extended Abstract, Lecture Notes in Computer Science (Proc. DIMACS Workshop Graph Drawing, 1994), R. Tamassia and I. G. Tollis, eds., vol. 894, Springer-Verlag, 1995, 159-166.

3. ___ Rectangle-visibility representations of bipartite graphs (submitted).

4. M. Gardner, Mathematical Games, Scientific American 242 (Feb. 1980) 14-19.

5. M. R. Garey, D. S. Johnson, and H. C. So, An application of graph coloring to printed circuit testing, IEEE Trans. Circuits and Systems CAS-23 (1976) 591-599.

6. J. P. Hutchinson, Coloring ordinary maps, maps of empires, and maps of the Moon, Math. Mag. 66 (1993) 211-226.

7. J. P. Hutchinson, T. Shermer, and A. Vince, On representations of some thickness-two graphs (submitted).

8. D. G. Kirkpatrick and S. K. Wismath, Weighted visibility graphs of bars and related flow problems, Lecture Notes in Computer Science (Proc. Ist Workshop Algorithms Data Struct.), vol. 382, Springer-Verlag, 1989, 325-334.

9. A. Mansfield, Determining the thickness of graphs is NP-hard, Math. Proc. Cambridge Phil. Soc. 93 (1983) 9-23.

10. H. Meijer, personal communication.

11. J. O'Rourke, Art Gallery Theorems and Algorithms, Oxford University Press, N.Y., 1987.

12. G. Ringel, Färbungsproblems auf Flächen und Graphen, Deutscher Verlag der Wissenschaften, Berlin, 1959.

13. E. Steinitz and H. Rademacher, Vorlesungen über die Theorie der Polyeder, Springer, Berlin, 1934.

14. R. Tamassia and I. G. Tollis, A unified approach to visibility representations of planar graphs, Disc. and Comp. Geom. 1 (1986) 321-341.

15. C. Thomassen, Rectilinear drawings of graphs, J. Graph Theory 12 (1988) 335341.

16. W. T. Tutte, On the non-biplanar character of the complete 9-graph, Canad. Math. Bull. 6 (1963) 319-330.

17. ___ The thickness of a graph, Indag. Math. 25 (1963) 567-577.

18. J. D. Ullman, Computational Aspects of VLSI Design, Computer Science Press, Rockville, Md., 1984.

19. S. K. Wismath, Characterizing bar line-of-sight graphs, Proc. 1st Symp. Comp. Geom., ACM (1985) 147-152. 